Meta

Journal des traducteurs

Translators' Journal

\title{
Table ronde sur l'évolution de la traduction
}

\section{Jean-Paul Coty, Robert Dubuc, Mary Plaice, Gisèle Poisson et Jacques Poisson}

Volume 20, numéro 1, mars 1975

Vingt ans de traduction. Bilan et prospectives

URI : https://id.erudit.org/iderudit/002412ar

DOI : https://doi.org/10.7202/002412ar

Aller au sommaire du numéro

Éditeur(s)

Les Presses de l'Université de Montréal

ISSN

0026-0452 (imprimé)

1492-1421 (numérique)

Découvrir la revue

Citer cet article

Coty, J.-P., Dubuc, R., Plaice, M., Poisson, G. \& Poisson, J. (1975). Table ronde sur l'évolution de la traduction. Meta, 20(1), 58-70. https://doi.org/10.7202/002412ar d'utilisation que vous pouvez consulter en ligne.

https://apropos.erudit.org/fr/usagers/politique-dutilisation/ 


\section{Table ronde sur l'évolution de la traduction}

Nous avons réuni autour d'une même table des traducteurs aux expériences diversifiées afin de sonder ce que sera la traduction demain. $\mathbf{M}^{\text {me }}$ Mary Plaice, traductrice au ministère des Communications du gouvernement du Québec, a bien voulu se faire le porte-parole des traducteurs anglophones; $M^{\text {lle }}$ Gisèle Poisson a consenti à nous sacrifier quelques heures de son indépendance si chèrement acquise pour nous faire partager la richesse de son expérience. Quant à M. J.-P. Coty, qui rayonne maintenant du Conseil de la Fédération internationale des traducteurs, il a accepté, avec toute la verve qu'on lui connaît, de nous éclairer du feu d'artifice de ses idées jaillissantes. À cela, il ne manquait que la sage réflexion d'un traducteur solitaire qui s'est depuis le début de sa carrière initéressé à la nature de la traduction et à son influence sociale, Jacques Poisson, traducteur-conseil, qui nous a fait l'honneur de se joindre à nous. M. Lucien Forgues, vice-chargé d'études au Service de linguistique de la Sun Life du Canada, a été empêché de participer à nos délibérations à cause d'un deuil dans sa famille. Son absence a été regrettée de tous.

Il ne me reste qu'à remercier les participants de leur si agréable collaboration. Je regrette de n'avoir pu restituer dans ce compte rendu l'humour pétillant qui a jailli tout au long de ce débat, les contraintes d'espace nous obligeant à nous en tenir à l'essentiel.

Les initiales au début de chacune des interventions permettent d'en identifier l'auteur. La parole est à la tribune.

ROBERT DUBuC

R. D. - Depuis vingt ans, l'évolution de l'exercice de la profession a été marquée par l'accélération du débit, l'accroissement du caractère technique des traductions et une importance accrue accordée à la terminologie.

Peut-on prévoir que, pour les vingt prochaines années, ces tendances vont s'affirmer et dans quelle mesure?

G. P. - L'accroissement quantitatif, à n'en pas douter. Mais la traduction n'a-t-elle pas toujours été, ici du moins, très nettement orientée vers la technique? 
R. D. - Pour ce qui est du Gouvernement fédéral, c'est la traduction parlementaire qui a longtemps représenté l'essentiel du travail du Bureau des traductions. Aujourd'hui, elle n'en représente plus qu'une partie largement débordée par les travaux techniques des ministères.

J.-P. C. - Ces trois questions me paraissent étroitement liées. En effet, l'accroissement de la demande amenée d'une part par l'augmentation de la production de textes et l'extension du public de langue étrangère et, d'autre part, en ce qui concerne le Québec, par l'adoption de la loi 22 instituant le français langue officielle, entraîne l'augmentation du nombre de textes à traduire; cette augmentation touche nécessairement les textes techniques. Prenons un exemple; il y a dix ans, pour les instruments médicaux, la traduction d'un mode d'emploi d'une dizaine de lignes suffisait, aujourd'hui, c'est un manuel de 200 à 300 pages qu'on nous demande de traduire. On voit donc comment l'accroissement quantitatif nous entraîne dans des textes d'un caractère de plus en plus technique. Dans cette optique, on voit sans peine comment la terminologie revêt une importance accrue. L'exactitude devient une nécessité impérieuse.

M. P. - Pour les traducteurs anglophones, le fait qu'on rédige beaucoup plus en français augmente considérablement le volume des traductions à faire. Prenons par exemple, au Gouvernement fédéral, alors qu'il y a dix ans les traductions vers l'anglais ne comptaient que pour $5 \%$ de l'ensemble, aujourd'hui, elles comptent pour $20 \%$ et dans les quinze prochaines années, cette proportion pourrait atteindre $40 \%$ et même davantage.

J.-P. C. - C'est aussi le cas dans l'entreprise privée, étant donné que, maintenant, tous les textes gouvernementaux sont communiqués en français aux entreprises.

J. P. - Justement, il me semble que le problème de l'orientation future de la traduction comporte une dimension politique qu'on ne saurait escamoter. En effet, le Québec connaît un bipartisme axé sur les options constitutionnelles, ce qui est nouveau pour lui. À l'intérieur du mouvement indépendantiste, on peut discerner deux courants bien nets : l'un francophile, l'autre francophobe. Dans l'hypothèse du triomphe de l'aile francophobe - tendance renforcée par la défrancisation des couches jeunes de la population soumise à un enseignement « américain », on peut prévoir une sorte d'annexion culturelle aux États-Unis qui modifierait profondément les données de l'exercice de la profession.

Quant à la terminologie, elle est promise à un progrès considérable, chacun étant sensibilisé aux problèmes énormes qu'elle pose aux traducteurs. La Banque de terminologie de l'Université de Montréal a amorcé un mouvement de solution qui me paraît irréversible.

M. P. - Pour les traducteurs anglais, il se pose des difficultés particulières du fait que les recherches terminologiques ont été orientées de l'anglais vers le français. Des ouvrages comme le Bélisle technique, les cahiers de l'O.L.F., les vocabulaires du Secrétariat d'État, ne permettent pas toujours l'utilisation du français vers l'anglais.

G. P. - Sauf pour les derniers cahiers de l'Office. 
M. P. - Cette situation crée parfois des problèmes énormes aux traducteurs anglophones.

R. D. - Si l'évolution que vous nous avez annoncée tout à l'heure se réalise, il ne fait pas de doute que la situation va se redresser. D'ailleurs, les techniques d'utilisation des fichiers automatiques permettent la réversibilité totale de la matière emmagasinée. C'est de là que peut venir la solution.

Je voudrais poser une question à M. Coty. Comment l'évolution que vous avez si bien caractérisée va-t-elle influer sur la formation des traducteurs ? Un traducteur généraliste sera-t-il en mesure de faire face à la situation ? Ou devra-t-on exiger du futur traducteur qu'il prenne d'abord un grade dans une spécialité technique avant de venir faire ses études en traduction?

J.-P. C. - Le traducteur généraliste ne peut plus faire face à la demande. Un généraliste ne peut traduire, au pied levé, un manuel d'électrocardiographie.

G. P. - Ce n'est pas nouveau, cela ; il en a toujours été ainsi. Cela tient à la traduction.

J.-P.C. - Ce qui est nouveau, c'est le niveau de technicité des textes à traduire. Nous tombons dans la technique pure. Il faut des traducteurs spécialisés, quelles que soient les prétentions contraires qu'on puisse avoir, par exemple, au niveau du Bureau des traductions du Gouvernement fédéral.

J. P. - Et, tres souvent, un texte spécialisé déborde sur un grand nombre de disciplines. La difficulté alors devient insurmontable.

M. P. - Ne serait-il pas possible de recourir alors à des équipes mixtes de spécialistes et de traducteurs ?

J.-P. C. - Là, on vit un très beau rêve ! S'il fallait que je dise à mon employeur, il me faut, pour traduire, un médecin, un biologiste, un terminologue, on me dirait poliment que la question sera étudiéc en Comité. Alors là, on sait ce qu'il en adviendrait.

R. D. - Mais, au niveau de la formation même du traducteur, estimez-vous qu'on doive former un généraliste apte à se spécialiser ou mettre sur le marché du travail un spécialiste.

G. P. - Il faut former un généraliste apte à se spécialiser.

J.-P. C. - Je suis d'accord. Il faut que le traducteur « ait des clartés de tout». Si vous en faites un spécialiste au départ, je crois que c'est une erreur.

G. P. - Les risques sont grands qu'il n'arrive pas à manier la langue avec toute la rigueur qu'exige un bon métier.

M. P. - Il faut beaucoup de culture, en particulier la pratique de la rédaction.

J.-P. C. - L La grande lacune des jeunes traducteurs, c'est la culture générale. La traduction technique est toujours multiforme; la spécialisation immédiate ne peut conduire qu'à la catastrophe. 
R. D. - Pour revenir à l'aspect mis en évidence par M. Poisson, peut-on se demander quel serait le visage de la traduction dans un Québec indépendant ?

G. P. - Il y a si longtemps qu'on laisse les autres penser à notre place, qu'on va encore continuer à le faire pendant un bon bout de temps.

J. P. - S'il y a un courant annexionniste, la nécessité de la traduction pourrait bien être éliminée.

J.-P. C. - Il y aurait aussi un plus grand besoin de se définir. L'effort de création serait nécessairement plus grand.

M. P. - Il y aura quand même toujours besoin de traducteurs vers l'anglais !

R. D. - Vous n'êtes donc pas menacés!

R. D. - Depuis vingt ans, on a accordé plus d'importance à la formation du traducteur. Des écoles professionnelles de niveau universitaire ont été mises sur pied et, de plus en plus, on exige du traducteur qu'il ait reçu une véritable formation professionnelle. Par contre, on assiste à l'heure actuelle à une désaffection presque totale du système scolaire à l'égard de l'enseignement systématique de la langue maternelle. Comment peut-on prévoir que ces deux données, en apparence contradictoires, vont influer sur l'exercice de la profession?

- Comment vont réagir les écoles de traduction?

- Comment va réagir le marché ?

J. P. - Je proposerais aux écoles de traduction une sélection extrêmement rigoureuse des candidats. Je sais que cette exigence va à l'encontre des tendances actuelles des universités, qui sont plutôt «laxistes ». Nous aboutissons donc à une impasse. La démocratisation de l'enseignement — si nécessaire et si impérieuse s'est malheureusement accompagnée d'une démagogie de la facilité qui joue contre la discipline nécessaire à l'apprentissage du français. Je me demande s'il est possible à une école de traduction dans le cadre universitaire de sortir de ce dilemne et s'il n'y aurait pas lieu de fonder, hors de l'université, une école vraiment capable de dispenser l'enseignement de la traduction. C'est peut-être utopique, mais je me demande si le Gouvernement fédéral, sous réserve des accommodements constitutionnels nécessaires, ne pourrait pas prendre une telle école à sa charge ? Tout cela reste à explorer pour sortir de l'impasse.

R. D. - Une école privée subventionnée serait-elle viable?

J.P. - J'aurais confiance qu'une telle formule élimine les servitudes d'un appareil administratif énorme et qu'elle permette d'innover. Le tout serait de savoir d'où viendrait le financement.

M. P. - Pour les traducteurs anglophones, les programmes de formation sont nettement insuffisants. Il y a aussi le problème de la langue de travail. Nous nous retrouvons en traduction comme les Canadiens français dans le milieu des affaires. Il nous faut posséder le français. Une connaissance passive de cette langue ne 
suffit pas. Les possibilités d'avancement sont extrêmement minces. Il faut que les traducteurs anglophones dépensent le plus clair de leurs énergies à acquérir le français plutôt qu'à se perfectionner dans leur langue. Ils sont de ce fait en butte aux gallicismes et la qualité de leurs moyens d'expression s'en trouve altérée. Le nouveau programme de maîtrise à l'Université de Montréal améliore la situation, mais beaucoup reste à faire.

En ce qui concerne l'enseignement de la langue maternelle, il y a un revirement de la situation. On est, dans le secteur anglophone, de plus en plus conscient de son importance.

Il reviendrait aux sociétés professionnelles de traducteurs d'intervenir auprès du ministère de l'Éducation pour que soit revalorisé l'enseignement de la langue maternelle.

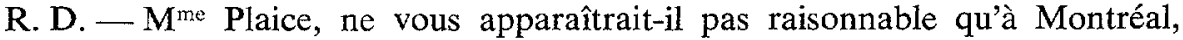
les universités anglophones, McGill et Sir George Williams, prennent en charge la formation des traducteurs anglophones?

M.P. - Je suis tout à fait d'accord. Peut-être que notre équipe au ministère des Communications pourrait aider à la mise sur pied d'un tel programme de formation?

J.-P. C. - En tant que professeur à l'École de traduction, je me rends compte jusqu'à quel point la méconnaissance de la langue maternelle, jönte à l'ignorance des principes élémentaires de la rédaction, nuit à la qualité de la traduction. Les écoles de traduction ne devraient accepter que des candidats possédant une connaissance plus que suffisante de la langue maternelle. Il faudrait exiger que les futurs traducteurs soient avant tout des rédacteurs. Il faut se méfier des «bilingues ». M. Grandjouan donne une excellente définition de ce qu'exige la traduction : «Dire bien, dans une langue qu'on sait très bien, ce qu'on a très bien compris dans une langue qu'on sait bien. » Il faudrait sélectionner les candidats d'après leur style plutôt que d'après leurs erreurs d'interprétation. On peut combattre l'erreur, mais difficilement le charabia.

Si l'université ne répond pas à ces exigences, il faudrait songer à la proposition d'une école privée, mise de l'avant par M. Poisson. Il y a une école privée toute trouvée, c'est la Société des traducteurs du Québec, qui a tous les pouvoirs en ce sens, à condition, bien entendu, que l'Université ne veuille pas changer d'attitude, ni tenir compte de ces exigences. La S.T.Q. a déjà ses cours de perfectionnement, elle pourrait s'organiser pour dispenser des cours de formation.

\section{J. P. - Pourrait-elle réagir contre l'idéologie du « laxisme »?}

J.-P. C. - À condition d'avoir la reconnaissance professionnelle. Un des avantages de la reconnaissance, c'est de pouvoir appliquer des normes : n'accepter dans la Société que les personnes qui savent s'exprimer correctement.

La réaction du marché diffère selon qu'on se trouve dans le secteur public ou le secteur privé. Dans le secteur public, l'employeur est en mesure d'évaluer le candidat qui se présente. Dans le secteur privé, en règle générale, l'employeur est 
dans l'impossibilité d'apprécier la valeur ou les lacunes d'une traduction. C'est seulement la reconnaissance professionnelle qui pourra permettre à l'entreprise privée de guider son choix. Les employeurs s'en remettront à la sanction officielle du gouvernement pour, au moins, s'éviter des ennuis.

J. P. - Il faudrait aussi distinguer entre les traductions symboliques et les traductions utilitaires. Au Gouvernement fédéral, les deux tiers des traductions sont symboliques.

G. P. - Il en est de même dans l'entreprise.

J.P. - Comment voulez-vous que celui qui commande une traduction symbolique s'intéresse à la qualité du produit fini?

G. P. - Au sujet de la qualité du produit, vous me permettrez de faire appel à mon expérience personnelle. Tant que j'ai travaillé dans des entreprises anglophones et qu'on m'a laissé autorité sur mon travail, il m'a été possible de garantir la qualité du produit. La situation s'est gâtée à partir du moment où des cadres ou des spécialistes canadiens-français, formés aux Etats-Unis, ont voulu intervenir pour imposer «leur» mode d'expression. Le traducteur peut difficilement tirer son épingle du jeu dans ces circonstances.

J.-P. C. - Il est très fréquent qu'on vérifie la qualité d'une traduction auprès du personnel de la chaîne de conditionnement !

J. P. - C'est une question de normes. Il faudrait faire accepter une autorité normative ; c'est l'orientation que devrait prendre notre profession.

R. D. - On rejoint alors M. Coty, lorsqu'il dit que la clé de la qualité de l'exercice de la profession tient à la reconnaissance professionnelle.

J.-P. C. - J'en suis convaincu. Nous aurons à ce moment une autorité qui pourra s'imposer. Avec l'exclusivité du titre et, peut-être même, la quasi exclusivité de l'acte, la Société professionnelle aura tous les pouvoirs pour exiger les normes de qualité qu'elle juge essentielles.

J.P. - Dans notre profession, nous nous trouvons dans la situation du chirurgien qui se ferait indiquer par la famille de son patient les points d'incision, les organes à enlever...

M. P. - Pour revenir à l'idée d'une école de traduction indépendante, avant de songer à la fonder, ne vaudrait-il pas mieux faire en sorte que les sociétés professionnelles aient leur mot à dire sur les critères d'admission aux écoles de traduction? Pouvoir, par exemple, imposer à certains candidats une année de propédeutique comprenant des lectures, des matières de culture générale et la pratique de la rédaction.

R. D. - Si, en théorie, la collaboration entre la profession et les universités est un principe admis par tous, en pratique, les modalités d'une collaboration étroite semblent difficiles à définir. Les universitaires sont plutôt jaloux de leurs prérogatives. 
G. P. - Il me scmble que les écoles de traduction dans les universités essaient de faire des virtuoses de la traduction avant de former de simples traducteurs. Les matières d'enseignement semblent destinées à des gens qui ont déjà une formation solide.

J.-P. C. - Cela dépend à quel niveau. L'amélioration par excellence a bien été d'amener des traducteurs professionnels à enseigner la traduction.

R. D. - Beaucoup d'efforts ont été faits pour rapprocher les programmes de formation des conditions d'exercice de la profession. Par exemple, le programme de la première année du baccalauréat vise à l'acquisition des rudiments du métier. Le malheur est que les carences des candidats sont telles qu'il faudrait beaucoup plus de temps qu'on en a pour pouvoir inculquer efficacement les connaissances suffisantes. Il en sera de même si l'on va chercher dans les autres facultés des spécialistes pour en faire des traducteurs. Il faudrait leur dispenser un programme de formation linguistique échelonné sur trois ou quatre ans avant de pouvoir en faire des traducteurs efficaces.

J. P. - Tout cela revient à poser le problème de la pédagogie de la traduction. On n'a pas encore établi de méthodologie, de pédagogie.

R. D. - La loi 22 , comme on l'a souligné, va créer une sorte de recours massif à la traduction; par contre, de plus en plus s'affirme chez les francophones le désir de s'exprimer, de créer dans leur langue, sans en être réduits exclusivement au rôle de traducteurs de la pensée d'autrui. N'y a-t-il pas risque, d'une part, que le sain exercice de la profession soit compromis par cette croissance quasi cancéreuse du débit de travail ? D'autre part, le traducteur doit-il prévoir la possibilité d'élargir ses attributions pour toucher aussi à la rédaction? Selon quelles modalités ? Comment se situe, dans cette perspective, la traduction vers l'anglais ?

J.P. - Cette question nous met en présence d'un danger: la saturation par la traduction. Au-delà d'un certain seuil, la présence de la traduction tend à détruire la langue d'arrivée. On connaît les risques d'interférences qui ont été abondamment étudiés, mais on s'est moins arrêté à l'appauvrissement du français par l'absence d'utilisation des ressources qui ne sont pas suggérées par l'anglais. De même, faute d'un ressourcement original, les références culturelles qui soustendent la vie d'une langue finissent par s'estomper, cc qui aboutit à une langue sans racine, artificielle, comme le latin au Moyen Âge.

Il faut tenir aussi compte du climat idéologique. La vie d'une langue doit être soutenue par un certain nombre d'idées positives à son endroit. En ce moment au Québec, on a l'impression d'assister à la destruction, à l'assassinat d'une langue, parce que toutes les idées qu'on entretient à son endroit sont négatives : le français serait une langue infirme, impuissante et qui exigerait un nombre incroyable de mots pour le moindre énoncé. Cet état d'esprit résulte d'un climat idéologique malsain. 
La Société des traducteurs devrait s'intéresser à ce problème, car il risque, à plus ou moins longue échéance, de faire disparaitre la nécessité du français et, par le fait même, la nécessité de la traduction.

J.-P. C. - Cette description du problème m'apparaît très réaliste. Et je crois qu'effectivement, il y a là responsabilité de la Société des traducteurs. Mais on pose, encore ici, la nécessité de la reconnaissance professionnelle. Le jour où la Société des traducteurs l'aura obtenue, elle deviendra, en quelque sorte, maîtresse des destinées de la langue au Québec. Le traducteur mène un combat d'avant-garde. J'admire beaucoup l'Office de la langue française et son travail - c'est un peu comme l'Académie -, mais ses interventions ressemblent à celles des carabiniers : elles arrivent lorsque tout est fini. La Société des traducteurs devra prendre des décisions avant que les autres ne les aient prises. Sa responsabilité est liée aux pouvoirs qu'on voudra bien lui reconnaître. Il n'y aura pas de société de traducteurs reconnue tant qu'on n'aura pas reconnu la nécessité de porter des jugements sur la langue... pensée...

G. P. - Et sur les causes profondes de la mutilation de la langue et de la

J.-P. C. - On en arrive à constater l'importance croissante des traducteurs au sein de leur société ; l'influence qu'ils exercent sur la langue dépasse en importance celle des universités, parce qu'ils prennent des décisions que les universités ne feront que ratifier.

G. P. - La Société des traducteurs s'oriente-t-elle dans ce sens ?

J.-P. C. - J'ai tenté de lui donner cette orientation. Plus la Société prendra conscience de ses pouvoirs, plus elle prendra conscience de ses responsabilités. Plus elle prendra conscience de ses responsabilités, plus son rôle prendra une importance sociale en ce qui concerne la langue et l'usage. Le traducteur est toujours sur la ligne de feu. C'est lui qui, le premier, doit trouver le mot qu'il faut.

J. P. - Pour éviter la saturation, il n'y a qu'un moyen, c'est de stimuler la création de textes de source.

J.-P. C. - Nous allons certainement aller, dans certains domaines, vers une rédaction totale en français.

J. P. - Peut-on prévoir que les traducteurs en viennent à recommander moins de traduction?

J.-P. C. - Certainement. Je cite ici le rapport Gendron, article 90, il propose : «de susciter la création, au niveau universitaire, d'une école francophone de publicité qui assurerait la formation professionnelle des publicitaires sur les plans commercial, technique et linguistique » et l'article 91 incite les agences de publicité à confier leurs campagnes de publicité en français à des publicitaires francophones et d'éliminer la traduction des textes anglais pour les remplacer par des créations en langue française.

M. P. - Je me demande s'il n'y aurait pas lieu tout simplement d'éliminer la traduction des textes de nature purement «informative» ou documentaire. N'y aurait-il pas moyen d'enseigner les deux langues avec suffisamment d'efficacité 
pour permettre à chacun de prendre connaissance d'un texte écrit dans sa langue seconde? On ne ferait alors traduire que les textes importants destinés à une large diffusion. Il faut absolument ne traduire que les textes qui en valent la peine.

R. D. - Au-delà d'un certain seuil quantitatif, en effet, la qualité n'est plus possible.

G. P. - Cette saturation est un élément destructeur de la langue.

J. P. - Si j'avais à détruire une langue, je me servirais de la traduction.

G. P. - Le grand danger qui guette le pigiste, c'est le risque de troquer la qualité pour la quantité.

J.-P. C. - Le pigiste pressé aura recours à l'« entre guillemets » pour tous les problèmes difficiles de traduction. Et ces « entre guillemets » vont finir par passer dans la langue.

G. P. - Mais les pigistes ne sont guère plus à blâmer que les autres. La même chose se passe dans les bureaux de traduction.

R. D. - Nous retrouvons le problème de la saturation soulevé par M. Poisson ; comment peut-on espérer d'une traduction faite à la chaîne, à un débit industriel, le moindre degré de qualité ? C'est toujours un sous-produit de l'esprit.

R. D. - La technique du $\mathrm{xx}^{\mathrm{e}}$ siècle permet d'utiliser la machine pour libérer le travailleur des tâches mécaniques, de façon qu'il puisse consacrer plus d'efforts aux aspects créateurs ou intellectuels de son travail. On sait que l'expérience de la traduction automatique s'est révélée décevante. Certaines formules de traduction, assistées par ordinateur, semblent prometteuses. Les banques de terminologie s'offrent pour résoudre l'épineux problème de la terminologie. Certains bureaux ont mis à l'essai des systèmes de machines à dicter, raccordées à un centre de dactylographie. Dans quelle mesure la profession doit-elle s'intéresser à ces innovations? Dans quelle mesure la formation du traducteur devrait-elle en tenir compte?

G. P. - Les banques de terminologie pourraient rendre d'immenses services, d'abord en évitant le dédoublement des recherches et les solutions contradictoires.

R. D. - L'expérience du Bureau fédéral des langues en Allemagne de l'Ouest est quand même significative. Les dirigeants de ce bureau estiment que l'automatisation de leur fichier terminologique leur a permis d'accroître des deux tiers le rendement quantitatif des traducteurs et la précision terminologique de $80 \%$.

J.-P. C. - Les avantages que l'ordinateur offre aux traducteurs sont inestimables. Je pense que trop de traducteurs en restent volontiers aux méthodes artisanales sans chercher d'autres solutions. Très peu de traducteurs, par exemple, travaillent au magnétophone. Pourtant, j'y vois un moyen de gagner beaucoup de temps. Il faudrait que l'étudiant s'initie au magnétophone. 
G. P. - Mais cette méthode exige un fonctionnement intellectuel accéléré et déjà le fonctionnement au ralenti est d'une grande pauvreté !

J.-P.C. - Il s'agit vraiment d'une technique nouvelle. La traduction au magnétophone livre un premier jet sur lequel ensuite on peut travailler. Les reprises sont plus faciles et cette technique permet une seconde version d'une excellente qualité. L'habitude y joue un rôle extrêmement important.

G. P. - Mais pour les textes difficiles... j'aurais peur d'une dégradation de la qualité.

M. P. - Nous avons fait l'expérience du magnétophone à la section française $\mathrm{du}$ bureau de Québec, au ministère des Communications et les résultats sont étonnants. La production s'est accrue de la moitié et la qualité était nettement supérieure à la production antérieure. Les expressions idiomatiques arrivent peutêtre plus spontanément en parlant qu'en écrivant.

J.-P. C. - J'allais le dire. Entendre la phrase qu'on dicte constitue un excellent contrôle. On s'entend dire "des imbécillités » et on peut alors se reprendre. C'est une méthode d'autocritique.

J. P. - La lenteur de la traduction écrite est peut-être un obstacle. Si l'on voulait marcher trop lentement, on risquerait de tomber.

R. D. - Il faudrait habituer les étudiants à travailler au magnétophone. Il semble y avoir là une méthode d'accélérer le débit sans nuire à la qualité.

M. P. - On l'a prouvé.

J.-P. C. - Il faut cependant des secrétaires d'excellente qualité. La secrétaire d'un bureau de traduction est, en quelque sorte, l'assistante du traducteur. La secrétaire doit s'habituer au débit, à la diction du traducteur; elle doit aussi s'habituer à la terminologie. Mais cela suppose aussi de la culture.

J. P. - Est-ce possible de trouver la perle rare?

J.-P. C. - Oui, à condition de la payer.

R. D. - Avez-vous autre chose à ajouter au sujet des outils mécaniques ?

J.-P.C. - Pour nous traducteurs médicaux, la Banque de terminologie ne peut pas être d'une grande utilité, parce que nous nous heurtons aux questions de recherche fondamentale où une théorie chasse l'autre. Nous sommes toujours en terrain vierge. Pour nous, les dictionnaires sont périmés, les manuels le sont également, même les glossaires le deviennent très vite. Comment trouver ailleurs une terminologie qui attend pour naître le bon vouloir du traducteur?

R. D. - Dans la mesure où vous pouvez transmettre à la Banque de terminologie ce que vous avez trouvé ou mis au point, ce pourra être utile à d'autres. Par contre, justice vous sera rendue lorsque vous aurez besoin de termes étrangers à votre spécialité. La Banque pourra alors vous être utile. En somme, une banque de terminologie devrait être une immense coopérative. 
J. P. - Comment arriver à une certaine unité du vocabulaire, si un Français, un Belge, ou un Suisse traduisent simultanément le même terme ?

J.-P. C. - La Fédération internationale des traducteurs pourrait en ce sens rendre d'immenses services en permettant l'intercommunication des groupes nationaux de traducteurs.

J. P. - Comment normaliser ?

J.-P.C. - Il faudrait recourir à un organisme de normalisation regroupant des spécialistes et des linguistes. Il faudrait aussi pouvoir disposer d'un organe de diffusion. C'est l'orientation que le Canada désirerait voir prendre à Babel, organe de la F.I.T.

R. D. - Il faut compter avec la facilité accrue des moyens de télécommunication. Les réseaux de communication entre les ordinateurs permettront instantanément la diffusion de l'information. Il y aura toujours, bien sûr, des obstacles d'ordre psychologique à vaincre...

M. P. - Mais il vaut la peine d'essayer de réussir cette unification, si vous ne voulez pas vous trouver, comme au Canada anglais, en perpétuel conflit entre deux normes d'usage : la britannique et l'américaine.

R. D. - Les associations professionnelles, c'est peut-être le fait saillant de la dernière décennie, ont connu un incontestable regain de vie, visant à donner à la profession les structures de base pour en assurer le sain exercice, tant pour le client que pour le traducteur. Peut-on prévoir comment vont désormais évoluer les sociétés professionnelles? Quels sont les risques de sclérose ? Comment pourront-elles conserver un dynamisme qui leur permette d'être à l'avant-garde des intérêts professionnels bien compris?

J. P. - Je me demande si, pour éviter la sclérose, les associations professionnelles ne devraient pas, à l'instar de l'Association des professeurs de français, donner une dimension sociale à leurs préoccupations, et non plus uniquement se soucier des intérêts pécuniaires de leurs membres.

M. P. - Plus je considère l'évolution des associations professionnelles, plus m'apparaît la nécessité pour la Société des traducteurs de fixer ses normes, de bien structurer son organisation pour bien jouer son rôle et ne pas tomber sous la coupe d'un contrôle extérieur. Une société trop étroitement contrôlée ne peut faire preuve de dynamisme.

Maintenant, je m'interroge sur la possibilité de fermer la profession. Je connais, par exemple, de nombreux écrivains anglophones qui font de la traduction littéraire. Allons-nous leur interdire de traduire?

J.-P. C. - Je suis tout à fait convaincu que l'avenir de la langue et l'avenir du traducteur reposent sur la reconnaissance de la Société. Cette reconnaissance ne saurait être un facteur de sclérose; au contraire, c'est alors que le traducteur 
prendra davantage conscience de sa responsabilité devant le public à l'égard de la langue. La sclérose c'est bien plutôt l'état actuel où nous nageons dans l'attente de la reconnaissance professionnelle.

Le dialogue avec l'employeur sera aussi modifié. Les représentants de la Société pourront parler beaucoup plus haut puisqu'ils feront partie d'un corps professionnel dûment reconnu.

C'est alors que la Société aura les moyens d'agir, qu'elle pourra le faire. Elle conférera alors au traducteur une juste fierté, lui, qui exerce, selon le mot de Grandjouan, "l'une des plus nobles des tâches serviles ».

M. P. - Mais les littéraires ?

J.-P. C. - Il faudrait qu'ils se joignent à nous, car ils ont aussi leur rôle à jouer. Nous les attendons.

G. P. - Vous ne pourrez jamais fermer la profession au point d'interdire à un écrivain de traduire une œuvre littéraire.

J.-P. C. - Telle n'est pas non plus notre intention. Mais nous avons besoin des traducteurs littéraires pour renforcer notre action linguistique.

G. P. - Ne nous heurtons-nous pas au mépris qu'au Canada français on entretient pour la qualité du français ? Si les membres de la S.T.Q. ne témoignent pas d'un grand respect pour leur langue, comment espérer attirer au sein de cette société des écrivains qui, eux, doivent l'avoir à un haut degré ?

J.-P.C. - Je pense que la S.T.Q. a déjà effectué une certaine épuration. Lorsqu'elle aura sa reconnaissance, la S.T.Q. pourra exercer une surveillance, et imposer des normes rigoureuses. La reconnaissance professionnclle va donner à la Société le sens de ses responsabilités.

M. P. - Mais elle l'a déjà. On n'admet plus « les gens qui s'intéressent à la traduction ». L'examen d'admission est rigoureusement corrigé.

J.-P. C. - L'avenir de la traduction, l'avenir et la défense de nos langues de travail, je les vois entre les mains des sociétés professionnelles. Les normes d'admission aux écoles professionnelles n'étant pas assez rigoureuses, les sociétés professionnelles doivent faire preuve d'une vigilance accrue.

G. P. - Je le veux bien, mais il faut tenir compte des difficultés énormes, inhérentes à l'exercice de la profession en milieu nord-américain. Ça demande presque de l'héroïsme pour conserver une certaine intégrité linguistique.

J.-P. C. - Je fais une grande confiance à la S.T.Q. pour le Québec, mais je crois que l'A.T.I.O. en Ontario et la C.T.I.N.B. pour le Nouveau-Brunswick pourront en faire autant. La seule façon de traiter les traducteurs, c'est de remettre leur sort entre lcurs mains.

G. P. - Prenons l'exemple de la traduction des lois. Elle est aussi mauvaise aujourd'hui qu'en 1950. Et les lois couvrent tous les secteurs de la vie du Canada français. 
M. P. - Je crois qu'il y a quand même eu amélioration.

G. P. - J'ai souvent besoin de consulter les lois. Chaque fois, les servitudes imposées sont épouvantables. Ce n'est pas du français.

J.-P.C. - Plus les sociétés auront de l'influence, plus elles seront en mesure de lutter contre un tel état de fait.

J. P. - À condition qu'elles sachent déborder les considérations stritement professionnelles. C'est une tâche difficile.

J.-P. C. - Cela dépend en grande partie du président. C'est aux traducteurs de le bien choisir.

R. D. - C'est aussi aux traducteurs de prendre leurs responsabilités. La Société ne sera toujours que ce que les traducteurs la feront. Il faut y jouer un rôle actif, si l'on veut qu'elle soit active et représentative.

J.-P. C. - Exact. Il ne faut pas avoir peur de prendre des responsabilités. Je voudrais ici rapporter une parole d'Edward Blake, citée par Keith Spicer: «On ne peut guère réaliser de grandes choses sans déranger quelqu'un ni quelque chose. »

R. D. - Cela nous fait une bonne conclusion. 